\title{
Severe clinical immunodeficiency in a patient with human immunodeficiency virus infection and relatively high CD4 counts: a case report
}

\author{
Mickael Essouma ${ }^{1 *}$, Larry N. Tangie ${ }^{1}$, Mazou N. Temgoua ${ }^{1}$, Ulrich Gabin Kenfack' ${ }^{1}$ Antonin N. Ndam¹,2 \\ and Celestin Danwang ${ }^{3}$
}

\begin{abstract}
Background: The coexistence of neuromeningeal cryptococcosis and Kaposi's sarcoma is not surprising in a patient with human immunodeficiency virus infection and a low CD4 count, although it is rarely described. However, we describe such an association in a patient with human immunodeficiency virus infection and a relatively high CD4 count.

Case presentation: A 41-year old Cameroonian woman presented to our hospital with subacute occipital headaches associated with photophobia, blurred vision, phonophobia, projectile vomiting, and tonic seizures. In her past history, there was an human immunodeficiency virus infection known for 12 years, for which she had been taking (with good compliance) tenofovir-lamivudine-efavirenz-based antiretroviral therapy for the same period of time. One month before the consultation, gastric Kaposi's sarcoma had been diagnosed, justifying the treatment with doxorubicin she had received. A clinical examination was unremarkable. A computed tomography scan of her brain was normal, and cerebrospinal fluid analysis revealed Cryptococcus neoformans. Her CD4 count was 353/mm³. Orally administered antifungal treatment with fluconazole (1200 mg/day) and flucytosine (1500 mg × 4/day) was started immediately, but she died on the sixth day of this treatment.
\end{abstract}

Conclusion: This clinical case shows that the coexistence of neuromeningeal cryptococcosis and gastric Kaposi's sarcoma is possible in all patients with human immunodeficiency virus infection, regardless of CD4 count.

Keywords: HIV infection, CD4 count, Immunodeficiency, Gastric Kaposi's sarcoma, Neuromeningeal cryptococcosis

\section{Background}

Since the introduction of highly active antiretroviral therapy (HAART) worldwide in 1996, there has been a decline in mortality from human immunodeficiency virus (HIV) infection. This is mainly due to a decrease in opportunistic diseases [1]. Neuromeningeal cryptococcosis is an opportunistic infection caused by a ubiquitous environmental en- capsulated yeast (Cryptococcus species), usually seen in patients with HIV infection and advanced immunodeficiency (CD4 count $<100$ cells $/ \mu$ l) [2]. Kaposi's sarcoma is another opportunistic disease associated with severe immunodeficiency, mostly occurring in individuals with HIV infection and a CD4 count $<100 \mu \mathrm{l}$, even though it can be seen at any stage of the disease [3].

Simultaneous diagnosis of neuromeningeal cryptococcosis and Kaposi's sarcoma is not surprising in a patient with HIV infection and low CD4 counts, although this is rarely reported. [4]. In this article, we describe the coexistence of neuromeningeal cryptococcosis and gastric Kaposi's sarcoma in a patient with HIV infection and a relatively high CD4 count, who had a fatal outcome. 


\section{Case presentation}

A 41-year-old Cameroonian woman was seen in a hospital for progressively worsening occipital headaches of 4 weeks' duration, associated with phonophobia, photophobia, blurred vision, projectile vomiting, and tonic seizures. In her past history, there was an HIV infection known for 12 years and for which she had been taking (with good compliance) tenofovir-lamivudine-efavirenzbased antiretroviral therapy for the same period. No opportunistic diseases had been noted in the last 11 years. One month prior to the consultation, an upper endoscopy (Fig. 1) performed to investigate persistent gastric pain led to the diagnosis of gastric Kaposi's sarcoma. She had already received a systemic chemotherapy cycle with doxorubicin. Upon admission, her clinical status revealed an ill-looking and alert (Glasgow Scale E4V5M6) patient. Her parameters were: temperature $37.8{ }^{\circ} \mathrm{C}$, blood pressure $176 / 120 \mathrm{mmHg}$, pulse rate 153 pulsations/minute, respiratory rate 25 cycles/minute, and weight $59 \mathrm{~kg}$. A computed tomography (CT) scan of her brain with injection of contrast products was normal. Cerebrospinal fluid (CSF) analysis revealed: protein 1.2 $\mathrm{g} / \mathrm{l}$, glucose $0.54 \mathrm{~g} / \mathrm{l}$ (concomitant glycemia $1.25 \mathrm{~g} / \mathrm{l}$ ), leukocyte $1 / \mathrm{mm}^{3}$, positive Indian ink stain and culture for Cryptococcus neoformans, and negative GeneXpert for acid-fast bacilli. Analysis of blood tests revealed: hemoglobin $9.6 \mathrm{~g} / \mathrm{dl}$, white blood cell count $2120 / \mathrm{mm}^{3}$, lymphocytes $848 / \mathrm{mm}^{3}$, platelets $604,000 / \mathrm{mm}^{3}, \mathrm{CD} 4$ count $353 / \mathrm{mm}^{3}$ (350-1600), C-reactive protein $48 \mathrm{mg} / \mathrm{l}$, blood urea nitrogen $0.15 \mathrm{~g} / \mathrm{l}$, and creatinine $7 \mathrm{mg} / \mathrm{l}$. Considering the diagnosis of neuromeningeal cryptococcosis, orally administered antifungal treatment with fluconazole $(1200 \mathrm{mg} /$ day $)$ and flucytosine $(1500 \mathrm{mg} \times 4 /$ day $)$ was started immediately. During hospitalization, her blood pressure normalized without treatment, but she died on the sixth day of antifungal therapy after a rapid deterioration of the state of conciousness.

\section{Discussion}

We describe the coexistence of neuromeningeal cryptococcosis and gastric Kaposi's sarcoma in a patient with HIV infection and a CD4 count of 353. These are two conditions that classify patients as having acquired immunodeficiency status of HIV infection, and therefore are often found in patients with low CD4 count. The major limitations of this work are the absence of concomitant HIV viral load which is the direct measure of HIV activity, and the lack of evidence of human herpesvirus 8 (HHV-8) infection.

Neuromeningeal cryptococcosis is the most common presentation of cryptococcal disease that classically develops in patients with HIV infection and CD4 counts $<100 / \mu \mathrm{l}[2]$. Other risk factors include organ transplantation, long-term corticosteroid use, chronic kidney and liver diseases, autoimmune/chronic inflammatory diseases, and idiopathic CD4+ lymphopenia. Since Cryptococcus species are optional intracellular germs, the increased risk of neuromeningeal cryptococcosis in these patients is mainly due to immunodeficiency affecting cellular immunity [5]. In fact, extrapulmonary infection with Cryptococcus species would be by an active mechanism of phagosomal extrusion, allowing them to spread from macrophage to macrophage. This hypothesis of spread of Cryptococcus species through macrophages is called the "Trojan horse" model, and the "Trojan horse" crossing of the blood-brain barrier is thought to be the main mechanism underlying neuromeningeal cryptococcosis. Beyond this general mechanism, other intrinsic factors and cryptococcal virulence may have been implicated in this patient. These intrinsic additive factors are essentially host molecules (protein CD44, protein kinase C, ganglioside GM1, and dual specificity tyrosine phosphorylation-regulated kinase 3 which is abbreviated to DYRK3) interacting with fungal ligands (for example, hyaluronic acid), while the virulence factors of Cryptococcus facilitating the crossing of the blood-brain barrier are cellular morphology (small cells) and the biochemical structure of

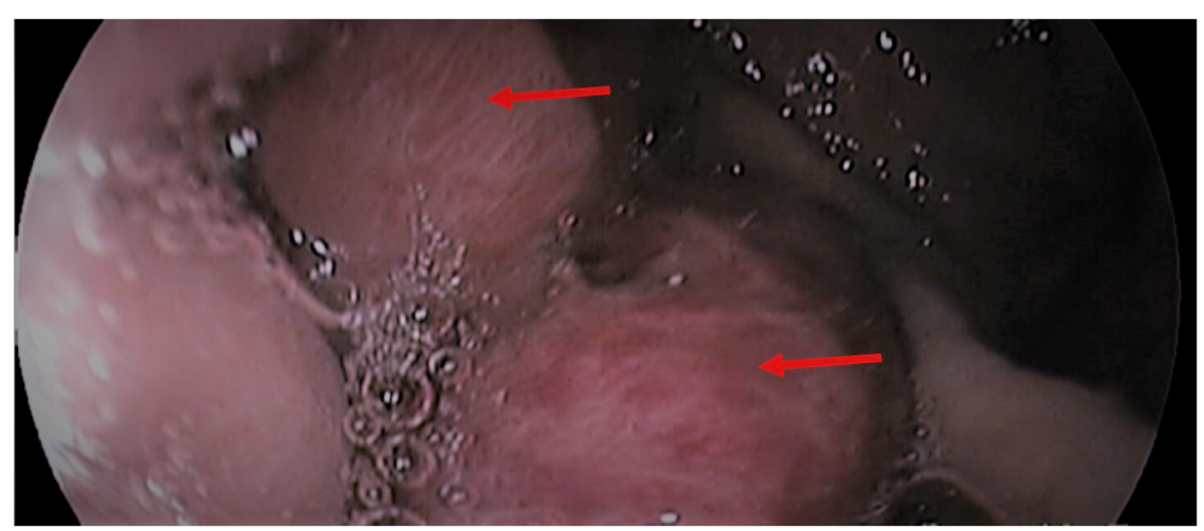

Fig. 1 Upper endoscopy showing infiltration of the gastric body by nodules of Kaposi's sarcoma (arrows) 
the capsule (presence of polysaccharides, mannitol, melanin, phospholipase, prostaglandins, and urease) [6].

As observed in this patient, the classic signs of meningitis are often absent during neuromeningeal cryptococcosis in a patient with HIV infection. Cryptococcal meningitis is typically a lymphocytic meningitis. However, this patient presented a poor CSF inflammatory response (1 leukocyte/ $\mathrm{mm}^{3}$ ) limiting conclusions in this regard. Of note, poor CSF inflammatory response is more frequent in patients with HIV infection and CD4 count $<50 / \mu \mathrm{l}$ [4]. Three molecules are recommended for the treatment of neuromeningeal cryptococcosis: amphotericin B, flucytosine, and fluconazole. This treatment takes place in three phases: induction $(2$ weeks), consolidation (8 weeks), and maintenance. During the induction phase, it is recommended to combine amphotericin B deoxycholate $(1 \mathrm{mg} / \mathrm{kg}$ per day) with flucytosine (100 mg/kg per day) for 1 week, followed by fluconazole (1200 mg/day for adults and $12 \mathrm{mg} / \mathrm{kg}$ per day for children and adolescents up to a daily dose of $800 \mathrm{mg}$ ) in monotherapy for 1 week [7]. In developing countries (including those in sub-Saharan Africa) where amphotericin B is not available, the best therapeutic alternative during the induction phase is the combination of fluconazole $(1200 \mathrm{mg} /$ day in adults and $12 \mathrm{mg} / \mathrm{kg}$ per day in children and adolescents up to a daily dose of $800 \mathrm{mg}$ ) with flucytosine $(100 \mathrm{mg} / \mathrm{kg}$ per day) for 2 weeks $[7,8]$. The choice of alternative treatment in this patient was based on this scientific evidence. Fluconazole is the only molecule recommended for the consolidation and maintenance phases of treatment.

Cryptococcal meningitis is associated with a bad prognosis, accounting for an estimated $15 \%$ of all deaths related to HIV infection globally, three quarters of which occur in sub-Saharan Africa [7]. Risk factors for mortality in patients with cryptococcal meningitis are: high CSF fungal load, CD4 count $<100$ cells/ $\mu$ l, poor CSF inflammatory response $\left(<20\right.$ leukocytes $\left./ \mathrm{mm}^{3}\right)$, and delayed diagnosis and treatment [4]. In untreated patients, further contributors to mortality are complications of raised intracranial pressure as well as immune reconstitution inflammatory syndrome associated with cryptococcal meningitis and HAART [7]. Although the patient did not have a low CD4 count and the antifungal treatment was administered promptly, the combination of other poor prognostic factors could, at least in part, explain the patient's death.

Kaposi's sarcoma is a malignant tumor of the vascular endothelium caused by the HHV-8 virus of the Herpesviridae family. This tumor can affect the skin and viscera; gastrointestinal involvement being the most frequent visceral involvement. Four clinical forms of Kaposi's sarcoma are described: (i) classical form (rare visceral involvement), (ii) endemic form (often found in Africa, mainly characterized by skin involvement), (iii) iatrogenic form (related to drug immunosuppression) and (iv) the epidemic form (related to immunosuppression by HIV, characterized by cutaneous involvement that may progress to visceral involvement at the late stage) [3]. Taking into account this classification, the most likely hypothesis was that of an epidemic Kaposi's sarcoma. What is surprising, however, is that our patient had a relatively high CD4 count and she had no cutaneous signs. Most patients have cutaneous signs and a CD4 count of less than 100 at the time of diagnosis of gastric Kaposi's sarcoma [3]. In such a patient with a relatively high CD4 count, the mechanisms that can explain Kaposi's sarcoma are, on the one hand, the interaction between HIV and HHV-8, and on the other hand, the interaction between HIV proteins (e.g. Tat) and host cell molecules (basic fibroblast growth factor, arginine-glycineaspartic acid binding integrins). These hypotheses assume evidence of HHV-8 infection and high HIV viral load, so increased viral activity [9]. The combination therapy regimen of HAART with doxorubicin that we used in this patient provides the best benefit in terms of survival for patients with gastric Kaposi's sarcoma but therapeutic failure is observed in the third of patients [3].

Co-occurrence of Kaposi's sarcoma and cryptococcal meningitis in the setting of HIV infection is rare, as illustrated by an unusual case of both diseases in a South African cohort of 127 individuals with HIV infection and low CD4 count [4]. The coexistence of both pathologies in this patient strongly suggested an acquired immunodeficiency syndrome, despite a relatively high CD4 count.

\section{Conclusions}

From this clinical case, there is evidence that both cryptococcal meningitis and gastric Kaposi's sarcoma can coexist at any stage of HIV infection, and irrespective of the CD4 count. A clinical suspicion for these diseases should still be maintained and a low CD4 count should not stop testing for the same.

\section{Abbreviations \\ CSF: Cerebrospinal fluid; HAART: Highly active antiretroviral therapy; HHV- 8: Human herpesvirus 8; HIV: Human immunodeficiency virus}

\section{Acknowledgements}

We thank Dr Atenguena Etienne who was part of the management team.

Funding

The authors did not receive any funding for this research.

\section{Availability of data and materials}

The authors declare that all data concerning this case report are provided within the manuscript.

\section{Authors' contributions}

Patient's management: LNT, MNT, UGK, ANN, ME, CD. Decision to submit the manuscript for publication: ME. Manuscript drafting: ME, MNT, LNT, CD, UGK. Manuscript revision: MNT, CD, LNT, UGK, ANN. All authors read and approved the final manuscript.

Ethics approval and consent to participate

Our institution does not require ethics approval for case reports. 


\section{Consent for publication}

Written informed consent was obtained from the next of kin of the patient for publication of this case report and any accompanying images. A copy of the written consent is available for review by the Editor-in-Chief of this journal.

\section{Competing interests}

The authors declare that they have no competing interests.

\section{Publisher's Note}

Springer Nature remains neutral with regard to jurisdictional claims in published maps and institutional affiliations.

\section{Author details}

'Department of Internal Medicine and Specialties, Faculty of Medicine and Biomedical Sciences, University of Yaounde I, Yaounde, Cameroon. ${ }^{2}$ Hepatogastroenterology Unit, Yaounde General Hospital, Yaounde, Cameroon. ${ }^{3}$ Department of Surgery and Specialties, Faculty of Medicine and Biomedical Sciences, University of Yaounde I, Yaounde, Cameroon.

Received: 4 September 2018 Accepted: 15 January 2019

Published online: 14 March 2019

\section{References}

1. Global Burden of Disease 2015 HIV collaborators. Estimates of global, regional, and national incidence, prevalence, and mortality of HIV, 19802015: the Global Burden of Disease Study 2015. Lancet HIV. 2016;3:e361-87.

2. Temfack E, Bigna JJ, Luma HN, Spijker R, Meintjes G, Jarvis JN, et al. Impact of routine cryptococcal antigen screening and targeted pre-emptive fluconazole therapy in antiretroviral naive HIV-infected adults with less than 100 CD4 cell counts: a systematic review and meta-analysis. Clin Infect Dis. 2018. In press; https://doi.org/10.1093/cid/ciy567.

3. Lee JA, Brenner L, Mourad B, Monteiro C, Vega KI, Munoz JC. Gastrointestinal Kaposi's sarcoma: case report and review of the literature. World J Gastrointest Pharmacol Ther. 2015;6(3):89-95.

4. Adeyemi BO, Ross A. Profile and acute mortality outcome of patients admitted with cryptococcal meningitis to an urban district hospital in KwaZulu-Natal, South Africa. S Afr Fam Pract. 2015;57(2):131-5.

5. Shribman S, Noyce A, Gnanapavan S, Lambourne J, Harrison T, Schon F. Cryptococcal meningitis in apparently immunocompetent patients: association with idiopathic CD4+ lymphopenia. Pract Neurol. 2018;18(2):166-9.

6. Liu TB, Perlin DS, Xue C. Molecular mechanisms of cryptococcal meningitis. Virulence. 2012;3(2):173-81

7. World Health Organization. Guidelines for the diagnosis, prevention and management of cryptococcal disease in HIV-infected adults, adolescents and children supplement to the 2016 consolidated guidelines on the use of antiretroviral drugs for treating and preventing HIV infection. 2018. Available from: https://www.who.int/hiv/pub/quidelines/cryptococcal-disease/en. Accessed 1 Sep 2018.

8. Molloy SF, Kanyama C, Heyderman RS, Loyse HA, Kouanfack C, Chanda D, et al. Antifungal combinations for treatment of cryptococcal meningitis in Africa. N Engl J Med. 2018;378:1004-17.

9. Barillari G, Sgadari C, Gendelman R, Morris CB, Palladino C, Nair BC, et al. The HIV-1 Tat protein promotes angiogenesis and Kaposi's sarcoma development by engaging RGD-binding integrins. [Abstract]. JAIDS. 1999;21(1):1.

Ready to submit your research? Choose BMC and benefit from:

- fast, convenient online submission

- thorough peer review by experienced researchers in your field

- rapid publication on acceptance

- support for research data, including large and complex data types

- gold Open Access which fosters wider collaboration and increased citations

- maximum visibility for your research: over $100 \mathrm{M}$ website views per year

At $\mathrm{BMC}$, research is always in progress.

Learn more biomedcentral.com/submissions 